\title{
Optimization of active distribution networks: Design and analysis of significative case studies for enabling control actions of real infrastructure
}

\author{
Diana Moneta ${ }^{1}$, Paolo Mora ${ }^{1}$, Giacomo Viganò ${ }^{2, a}$ and Gianluca Alimonti ${ }^{3}$ \\ ${ }^{1}$ RSE, Ricerca sul Sistema Energetico SpA, Italy \\ ${ }^{2}$ Università degli Studi di Milano \& RSE, Italy \\ ${ }^{3}$ INFN \& Università degli Studi di Milano, Italy
}

\begin{abstract}
The diffusion of Distributed Generation (DG) based on Renewable Energy Sources (RES) requires new strategies to ensure reliable and economic operation of the distribution networks and to support the diffusion of DG itself. An advanced algorithm (DISCoVER - DIStribution Company VoltagE Regulator) is being developed to optimize the operation of active network by means of an advanced voltage control based on several regulations. Starting from forecasted load and generation, real on-field measurements, technical constraints and costs for each resource, the algorithm generates for each time period a set of commands for controllable resources that guarantees achievement of technical goals minimizing the overall cost. Before integrating the controller into the telecontrol system of the real networks, and in order to validate the proper behaviour of the algorithm and to identify possible critical conditions, a complete simulation phase has started. The first step is concerning the definition of a wide range of "case studies", that are the combination of network topology, technical constraints and targets, load and generation profiles and "costs" of resources that define a valid context to test the algorithm, with particular focus on battery and RES management. First results achieved from simulation activity on test networks (based on real MV grids) and actual battery characteristics are given, together with prospective performance on real case applications.
\end{abstract}

\section{Introduction}

Reaching of the "20-20-20" targets on energy efficiency, greenhouse gases reduction, and Renewable Energy Resources (RES) exploitation is posing important challenges to the power systems; moreover in most EU countries the required contribution from the electricity sector will have to back-up the expected lower input from transportation and building sectors. The RES based generators are mainly connected to distribution networks (Medium Voltage-MV and Low Voltage-LV level), designed in a passive scenario with power flows from substations to users. A significant presence of distributed generators, especially in sunny rural areas with limited consumption, could cause severe problems on safe and reliable network operation, already explored into the literature [1].

\footnotetext{
${ }^{a}$ Corresponding author: giacomo.vigano@studenti.unimi.it
}

This is an Open Access article distributed under the terms of the Creative Commons Attribution License 4.0, which permits unrestricted use, distribution, and reproduction in any medium, provided the original work is properly cited. 
RES integration into the existing MV networks requires developing and testing innovative system concepts and technologies. In particular, a more effective functional contribution from DGs is expected, toward the so called "smart grid" paradigm where the Distribution System Operator (DSO) will have to properly balance the flows on its infrastructure relying on different regulation resources. The availability of several controls, owned by the DSO or made available by third-party resources, depicts a more complex scenario where a comprehensive optimum has to be identified.

\section{Optimization algorithm}

The proposed control approach relies on a technical-economic optimization procedure named DISCoVER (DIStribution Company VoltagE Regulator). Starting from forecasted load and generation, and considering technical constraints and dispatching costs for active and for reactive power, the proposed algorithm for each time period generates a set of commands (setpoint) for controllable resources that guarantees achievement of technical goals minimizing the overall dispatching cost. DISCoVER minimizes the total cost referred to the control strategies, even in presence of storage units, needed to satisfy the technical constraints. The optimization procedure relies on several resources with different "costs" that will be described in detail in Chapter 3.3.

\section{Case studies design}

In order to prepare an effective integration of the voltage control on the Supervisory Control And Data Acquisition (SCADA), several preliminary offline tests are carried out in smaller, but significant, test networks. These cases were designed in particular to first evaluate the algorithm behavior in different conditions, even in situations hardly replicable in the real field, second to tune the optimization parameters ("costs", etc.) to get a real feasibility of every set-point, with respect to the defined technical constraints. Finally the offline testing is carried out to check the correct information flow within the algorithm itself and with the boundary environment, including the calculation time.

Each case study is then composed by a combination of parameters, detailed in the following paragraphs, to highlight the previous goals.

\subsection{Test network}

The test network considered in this paper was designed starting from real MV networks (Fig. 1), in order to create a significant but realistic test bed. It consists of one HV/MV substation, $3 \mathrm{MV}$ feeders, and includes:

- 212 nodes, divided in $69 \mathrm{LV}$ nodes and $142 \mathrm{MV}$ nodes (MV/LV Substations, MV customers or transit nodes),

- a total load of $15.65 \mathrm{MW}$ from different customers (commercial, residential, industrial, and agriculture), among them 5.51 MW are directly connected to the MV level. The total power generation is $11.60 \mathrm{MW}$ divided in different type of resources, as summarized in the following Table 1.

Despite the nominal load is bigger than the total generation, the reverse power flow from the MV network to the HV network is very relevant because the network is mainly rural and the actual load is only a small fraction of the total nominal value. Conversely the generators, in particular the PV (photovoltaic) during the day, produce almost their maximum power. The reverse power flow may cause several problems, such as the possible overvoltage of portion of the network.

In the considered scenario the load and generation aren't equally distributed between the feeders, as usually occurs in real networks. The control system must then find a comprehensive solution that balances different network requirements. 


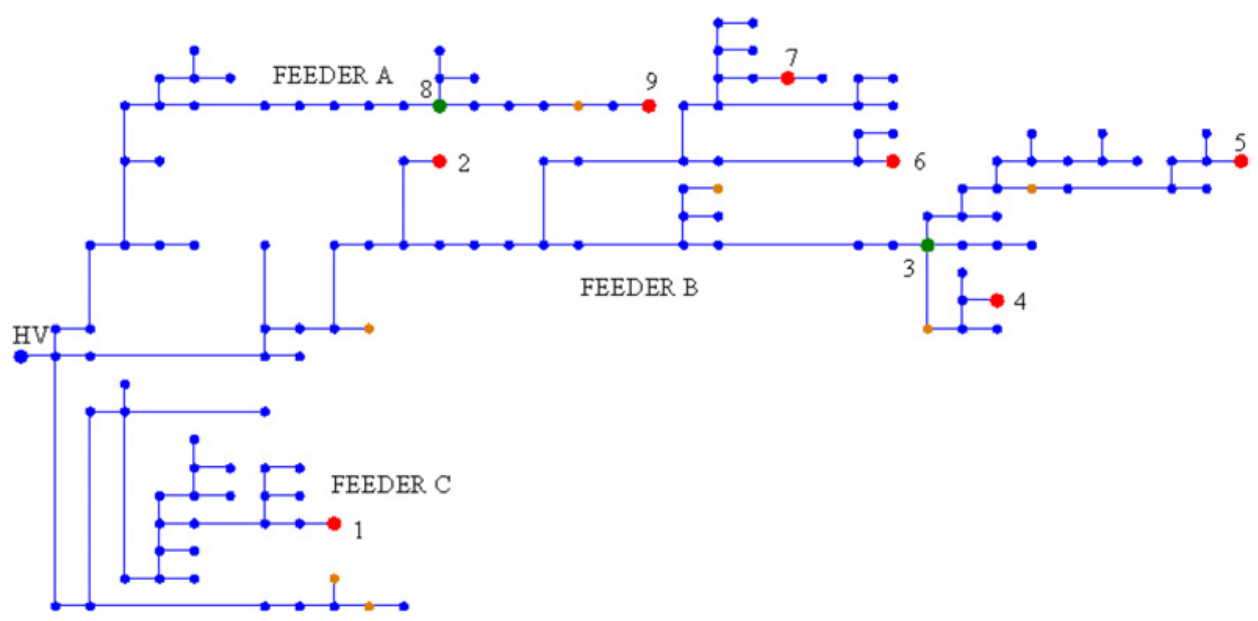

Figure 1. Test network. Only the MV nodes are showed. MV photovoltaic generators are red and other generators are orange. The green nodes represent the two possible positions for the storage (\#3 and \#8).

Table 1. Generators connected to the test network.

\begin{tabular}{|c|c|c|}
\hline Generators (number) & Type & Total power [MW] \\
\hline 2 & Hydroelectric & 0.86 \\
\hline 4 & Biomass & 2.26 \\
\hline 4 & others & 2.39 \\
\hline 9 & photovoltaic in MV & 3.96 \\
\hline$>30$ & photovoltaic in LV & 2.13 \\
\hline
\end{tabular}

\subsection{Technical parameters and constraints}

The network nominal voltage is $15 \mathrm{kV}$ for most of the MV nodes, while the voltage of the LV nodes is usually $400 \mathrm{~V}$ (quite representative of the Italian situation for rural areas). The desired voltage range is $0.96-1.05$ p.u. for the MV nodes, a usual voltage range for normal operating conditions. For the LV nodes the voltage range is larger: $0.9-1.1$ p.u. The algorithm is able to specify other voltage ranges according to the necessity of the network operation.

The thermal current limits vary in the network from 140 A to $330 \mathrm{~A}$, according to the type of cables (overhead or underground lines). However the current limits don't represent a problem in this network.

The impedances of the lines as seen from the HV node are not equal for all the feeders. The feeder C impedances are small, so the feeder voltage is always near the voltage of the MV bus-bar. Instead, the impedances of the other feeders are larger, so that small power flow can cause big voltage variations from the MV bus-bar.

According to the current Italian scenario, the reverse flow from MV to the HV level is not forbidden as a technical constraint in the problem. The only way to effectively prevent (or limit) the counterflow would be to use a storage with adequate capacity or to enable the active power modulation of generators or loads. Since the active power modulation is not considered in the current regulation and the storage capacity is too small (given the current CAPEX cost for this technology), the reverse power flow could be only decreased, setting a proper "costs" for the resources in the algorithm.

Other important parameters are the time horizon of the optimization and the length of time intervals in which the horizon is divided into. These parameters depend on the availability and reliability of the forecast and on the characteristic of the storage as it will be detailed in the next chapter. 


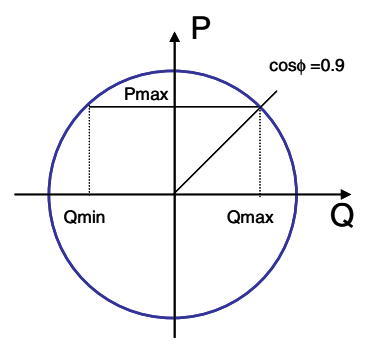

Figure 2. Example of simplified rectangular capability (photovoltaic generator).

\subsection{Regulation resources}

In the following test three available resources are considered.

a) OLTC: the on load tap changer can modify the transform ratio of a transformer. In the test network it is present only in the HV/MV transformer. In the reality the ratio assumes discrete values but in the present work the variation is considered as a continuous value in the range $-15 \div 15 \%$ of the nominal voltage.

b) $D G$ : distributed generators. Inverter base DG can, in principle, modulate both reactive and active power. The algorithm is able to include both the quantities but because the active power reduction entails an economic effect, given the incentives on the RES production, here only the reactive power is chosen for modulation by DG.

The number and type of available generators depend on the regulatory framework (for example all generators above a given size should provide certain network services), on the technical characteristics of the generators (PV are different from thermal units), and on the necessity of the network [3].

In this study all the MV photovoltaic generators are considered as controllable resources, since their power electronics make advanced control simpler and they represent the most numerous typology. According to the Italian connection rules [4] for LV and MV inverter based plants, the simplified capability of PV generators is rectangular (following scheme): for every value of active power the reactive power can vary in the interval $\pm \mathrm{P}_{\max } \cdot \tan [\operatorname{acos}(0.9)]$.

The only resources that can modulate their active power are the generators associated to the HV network (representing the prevailing network) and the storage.

c) Storage: the storage systems are very important element in the future grid, helping to increase the renewable penetration through the energy shift and other network support services. At the present state of art perhaps the batteries are still too expensive to allow long time energy shift. However a storage system can help to modulate the feeder voltage, to resolve short-term active congestion and to provide ancillary service. In this study the storage has the task to prevent voltage violation, reduce reverse power flow and reduce the losses.

To resolve the system where a storage unit with a recharge constraint is present, it is necessary to include an integral constraint in the problem. This involves the availability of reliable forecast data for the future periods to estimate the evolution of the system in term of load and generation behavior therefore identifying the best periods for the charge and the discharge of the battery. This increases the complexity of the system and introduces new variables: the length of the horizon and time intervals, the maximum and minimum charge of the battery and its initial and final charge levels.

The storage position is as important as its technical characteristics. For example if the storage is in the MV bus-bar, it will be used only for the energy, instead, in the farthest nodes of the network, it can be used also for the local voltage control. Moreover, if the chosen branch has an high generation, the active modulation is more important than the reactive modulation. There is no general rule to choose 
the proper position of the storage, adequate for the whole year. In the test case here analyzed the storage could be alternatively connected to the feeder A and B (Fig. 1) that present the bigger problems.

For simplicity only one storage unit is connected to the test network, with a typical capacity for centralised application on distribution networks of $2 \mathrm{MWh}$ and a rectangular capability of $\pm 1 \mathrm{MW}$ and \pm 1 MVAr. The network is analyzed with a time interval of one hour and a horizon of $24 \mathrm{~h}$; however these values are not fixed and the time horizon could be even longer. Nevertheless, lengthening the horizon could not improve the obtained solution because of the intrinsic lower reliability of RES based generation forecasts for distant events. A proper timing for real time optimization has to balance these conflicting aspects.

\section{4 "Costs" of regulation resources}

For every resource that exchanges power with the network a proper "cost" is set with respect to the reactive and active power injection and absorption. Since the regulation hasn't assigned yet the actual dispatching cost of each resource, the values here considered don't represent a real reward but a ranking criterion among resources that should be carefully identified because the calculated "optimum" state of the network depends strongly on them.

In the following the preliminary survey focused on identifying the proper balance between the different available resources is described. In the summarized simulations the costs of active power are greater than the reactive costs and the costs of all the resources and of the HV network are taken equal. With this configuration all the resources have the same economic importance, so their use depends mainly on the technical characteristic of the connection point. In this way the resources are equally used, respecting the constraints, to reduce the losses.

\subsection{Load and generation profiles}

Expected profiles for generation and load are important especially to determine the proper use of the storage. To remain close to reality, actual values of a real MV network were taken; in the real-time control these profiles come from the SCADA and are continuously updated thanks to weather forecasts and other dedicate tools. In particular the curve of the MV are real data while the profiles for the LV nodes are conventional and scaled to the nominal power of the network.

The profile changes greatly during the year, even if typical patterns recurring with small differences could be identified. In particular the profiles could be roughly grouped depending on the season (Winter, Spring/Autumn, Summer) and the day of the week (weekdays and weekend). Besides, particular conditions, for example cloudy day with low photovoltaic generation or other particular conditions of generation and load, should be explored.

\subsection{Summary of the test cases}

Next, three representative cases are analyzed:

1. A day with high generation and low load.

2. A day with low generation and high load.

3. A day with intermediate generation and load.

The first two cases represent extreme scenarios where the use of the control system is necessary to maintain stable the network. The last one is a day where the control system is not necessary, but it can improve the performance of the system. For each day few different tuned combinations of costs and resources are considered to assess their impact on the achievable results. 


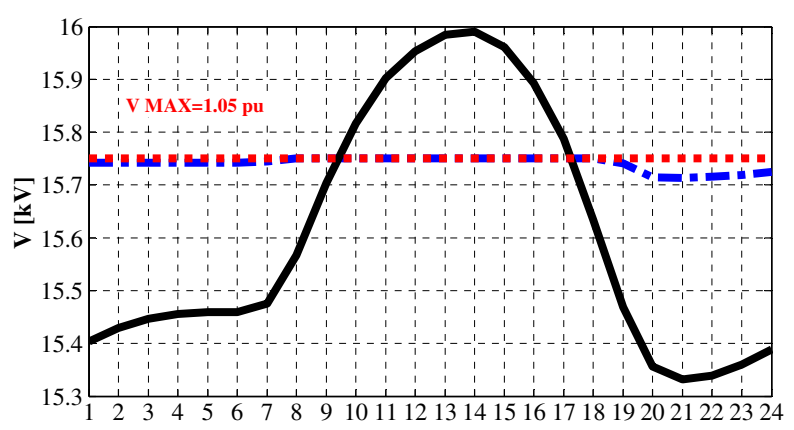

Figure 3. The voltage of the farthest nodes of feeder B as function of time. In black the values before the optimization and in dot-blue after the optimization. In red-dot the lower limit.

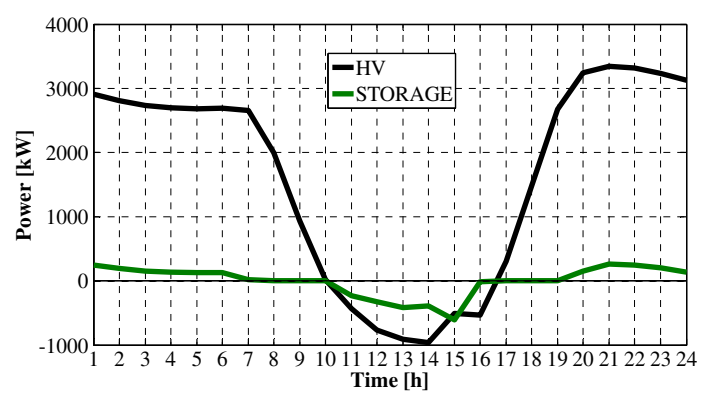

Figure 4. Power exchanged by the HV network (in black) and by the storage (in green). The storage decreases the reverse power flow and injects power during the load peak. The capacity of the storage isn't enough to absorb all the excess energy.

\section{Offline optimization results}

\subsection{Case 1}

In this case the load and generation curves are typical of a Summer Sunday: the load is mainly residential and the generation is almost all photovoltaic with a peak of about $5 \mathrm{MW}$. The production exceeds the load, with a medium value lower than $4 \mathrm{MW}$, in the central hours of the day, for a total energy of about 7.75 MWh. In the evening the generation goes near zero and there is the peak of residential load. This scenario is typical in the southern rural areas of Italy and its frequency is increasing.

In the baseline case (corresponding to the current operation on real networks) there are no resources available and the transform ratio of the transformer is fixed. Due to the high generation, the feeder B voltage goes above the upper voltage constraint in the central hours (Fig. 3) and the feeder A voltage goes below the lower constraint in the evening, when the load is rather high. In the second step, all the available resources previously described are activated. The storage is connected to the active feeder B and the set of costs is normal (Sect. 3.4).

In the calculated solution the OLTC keeps the bus-bar voltage high both in the morning and in the evening to sustain the voltage. The voltage is reduced during the reverse power flow to decrease the voltage of feeder B. In the central hours this action is not sufficient to prevent voltage violations: the generators in $\mathrm{A}$ and $\mathrm{C}$ thus inject reactive power to increase the voltage, the storage absorbs active power (Fig. 4) and the generators in B absorb reactive power to reduce the voltage (Fig. 5), closing the gap among the voltage profiles of the two feeders. With these new set-points the voltage in all the nodes of the network remains always in the chosen range. The injection of reactive power and the increase of the 


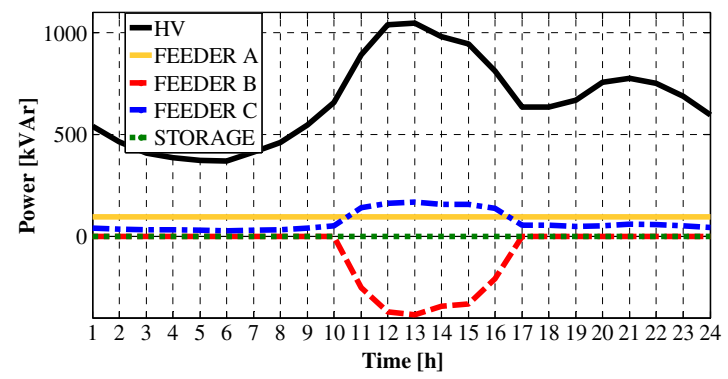

Figure 5. Reactive power as function of time for the different resources of the network in case 1 with normal costs.

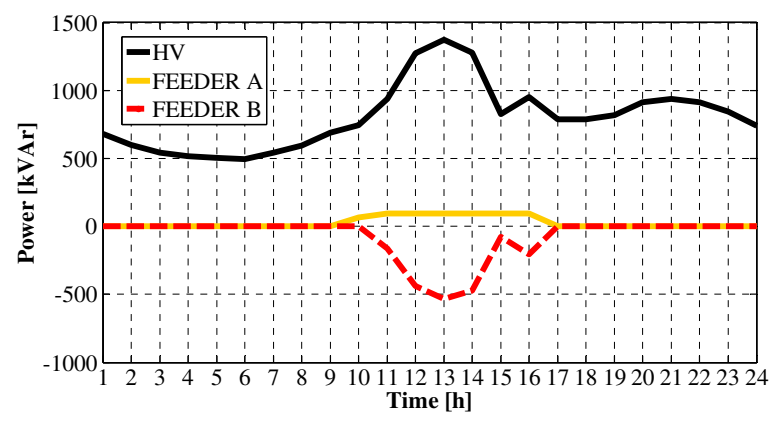

Figure 6. Reactive power as function of time for the different resources in case 1. Since the use of local resources is discouraged, the generators work only to respect the constraints (voltage, essentially).

voltage of the nodes are generally preferred by the algorithm because they indirectly decrease the losses. The reactive power injection reduces indeed the reactive power flow in the network (the generators are usually closer to the load than the HV network), and the voltage is raised to reduce the currents for the same power.

The use of the storage may reduce the losses as well, because storing the energy near the generators and loads, there is a lower total power exchange with the HV network. In this way the total losses decrease from $5,036 \mathrm{kWh}$ to $4,770 \mathrm{kWh}$.

Finally, the cost for the reactive modulation from the generators is increased. This condition makes the reactive modulation used only to prevent the voltage violation and not to reduce the losses (Fig. 6), so the total losses are $4,846 \mathrm{kWh}$, more than the previous case. In the same way the behavior of the storage can change with the variation of its costs.

\subsection{Case 2}

The reference day is now a winter cloudy day with very small production (less than $2 \mathrm{MW}$ ) and practically no photovoltaic contribution. The load is mainly industrial and has a peak value of about 5.5 MW. The load in this case is high so the power flow causes high voltage drops especially in the feeder A having a big impedance and the highest load. The voltage of feeder A may thus go below the lower limit during the hours with higher consumption (Fig. 7).

In the optimization procedure all the resources are activated and the storage is connected to the most problematic feeder A. To resolve the violations, the OLTC keeps the MV bus-bar voltage near its maximum value for all the time. Besides, the controllable PV generators and the storage produce reactive power to sustain the voltage (Fig. 8), keeping its value within the limits (Fig. 7). This action 


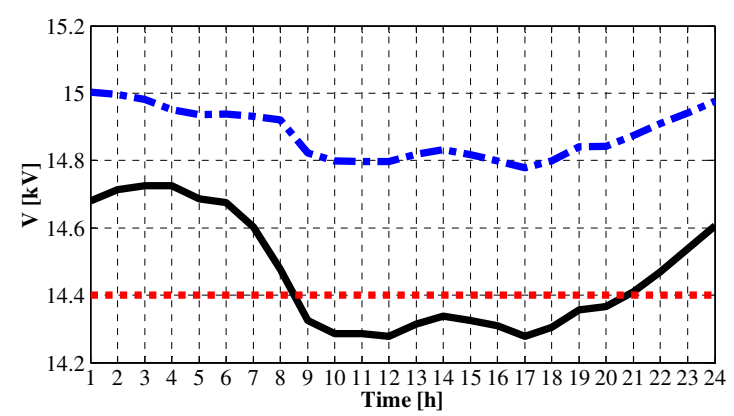

Figure 7. The voltage of the farthest nodes of feeder $\mathrm{A}$ as function of time. In black the values before the optimization and in dot-blue after the optimization. In red-dot is plotted the lower limit (0.96 p.u.).

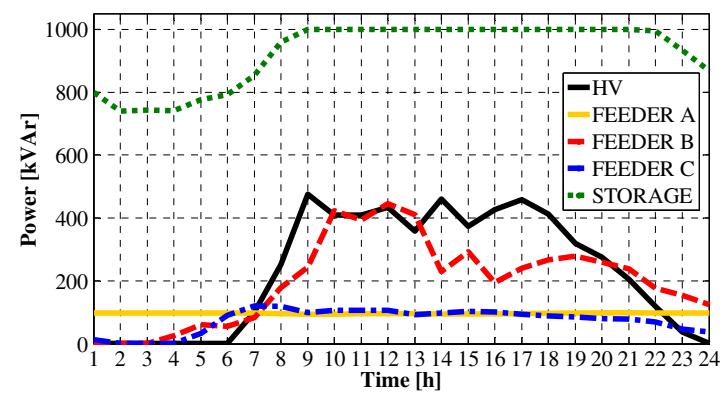

Figure 8. Reactive power as function of time for different resources of the network. In this case the load is always greater than the generation: all the DG inject reactive power.

has also the advantage to reduce the reactive flow in the network and then the losses, from $5,559 \mathrm{kWh}$ to $4,804 \mathrm{kWh}$. The storage doesn't exchange active power because there is no reverse power flow so that there is no surplus energy to conveniently store.

\subsection{Case 3}

It is now considered an average day, with a medium load of about $4 \mathrm{MW}$. The production, due to the photovoltaic generators, exceeds the load only in the central hours for a total energy of about $1 \mathrm{MWh}$. In this example the optimization is not strictly required because the voltage violations are negligible. In order to identify a "better" operation point, all the resources are made available and the storage is positioned in feeder A. In these normal operating conditions the algorithm permits to reduce:

- the active losses that go from $4780 \mathrm{kWh}$ to $4180 \mathrm{kWh}$;

- the reverse power flow to the HV network that goes from $800 \mathrm{kWh}$ to $65 \mathrm{kWh}$

- the reactive flow from the HV network, and thus to reduce the losses in the transmission network, that goes from $19800 \mathrm{kVArh}$ to 0.

It should be noted that these improvements are possible only with a particular set of costs: if the reactive modulation costs are too high, the DSO will not have any convenience to reduce the losses. The network behavior can thus strictly depend on the remuneration set by the regulations. 


\section{Final considerations and future steps of the activity}

The paper described a technical-economic optimization technique that could help DSOs in various critical situations in the operation of MV networks with significant presence of distributed generation. The procedure here proposed is already able to include storage units to help the voltage regulation and possible contingencies arising from an excess of energy from not programmable RES plants. A complete framework regarding the ancillary services reward offered by DGs is lacking and currently under discussion [5]; nevertheless this paper presented how different resource costs can effect the behavior of a real MV network.

The results here discussed show how an advanced control method could improve the system performances in terms of voltage quality service and economic benefit (losses reduction), both in critical and in normal operation conditions. In a growing RES scenario, the contribution from distributed generation, together with the use of storage unit, represents a precious option when the usual resources cannot guarantee an adequate operation on the whole network.

Finally, the calculation time of the optimization, requiring less than 10 seconds for 300 nodes on $24 \mathrm{~h}$ time horizon in $1 \mathrm{~h}$ step, appears adequate for the integration of the algorithm into the real time control infrastructure for large MV networks.

The authors thank Chiara Michelangeli and Claudio Carlini for their support in the simulation.

This work has been financed by the Research Fund for the Italian Electrical System under the Contract Agreement between RSE (formerly known as ERSE) and the Ministry of Economic Development - General Directorate for Nuclear Energy, Renewable Energy and Energy Efficiency stipulated on July 29th, 2009 in compliance with the Decree of March 19th, 2009.

\section{References}

[1] For a summary on possible impacts of distributed generation: CIGRE SC C6 (Distribution systems and dispersed generation) www. cigrec6.org

[2] D. Moneta, A. Gelmini, C. Carlini, M. Belotti, proceedings PSCC Power Systems Computation Conference, Stockholm, Sweden "Storage units: possible improvements for voltage control of MV distribution networks", August 22-26, 2011

[3] D. Craciun, D. Geibel, proceedings $22^{\text {nd }}$ Int. Conference on Electricity distribution CIRED 2013, "Evaluation of ancillary services provision capabilities from Distributed Energy Supply", Stockholm

[4] Comitato Elettrotecnico Italiano (CEI), norma 0-16:2012 "Reference technical rules for the connection of active and passive consumers to the HV and MV electrical networks of distribution Company", December 2012 (in Italian)

[5] Autorità per l'energia elettrica e il gas, DCO 354/2013/R/eel "Pubblico dibattito per la riforma delle modalità di approvvigionamento delle risorse per il servizio di dispacciamento, con particolare riferimento agli impianti di generazione distribuita e agli impianti alimentati dalle fonti rinnovabili non programmabili" (in Italian), August 2013, www . autorita.energia.it 